\title{
Schnyder Woods for Higher Genus Triangulated Surfaces*
}

\author{
Luca Castelli Aleardi $^{\dagger}$ \\ CS Department \\ Université Libre de Bruxelles \\ Bruxelles, Belgium \\ luca.castelli.aleardi@ulb.ac.be \\ Éric Fusy \\ Dep. of Mathematics \\ Simon Fraser University \\ Vancouver, Canada \\ eric.fusy@inria.fr
}

\author{
Thomas Lewiner \\ Dep. of Mathematics \\ PUC-Rio \\ Rio de Janeiro, Brazil \\ tomlew@mat.puc-rio.br
}

\begin{abstract}
Schnyder woods are a well known combinatorial structure for planar graphs, which yields a decomposition into 3 vertexspanning trees. Our goal is to extend definitions and algorithms for Schnyder woods designed for planar graphs (corresponding to combinatorial surfaces with the topology of the sphere, i.e., of genus 0) to the more general case of graphs embedded on surfaces of arbitrary genus. First, we define a new traversal order of the vertices of a triangulated surface of genus $g$ together with an orientation and coloration of the edges that extends the one proposed by Schnyder for the planar case. As a by-product we show how some recent schemes for compression and compact encoding of graphs can be extended to higher genus. All the algorithms presented here have linear time complexity.
\end{abstract}

\section{Categories and Subject Descriptors}

G.2.2 [Discrete Mathematics]: Graph Theory-Graph algorithms; E.4 [Data]: Coding and Information TheoryData compression and compaction

\section{General Terms}

Algorithms

\section{Keywords}

Schnyder woods. Triangulations. Higher genus surfaces. Graph encoding.

\section{INTRODUCTION}

Schnyder woods are a nice and deep combinatorial structure to finely capture the notion of planarity of a graph. They are named after W. Schnyder, who introduced these structures under the name of realizers and derived as main

\footnotetext{
* This work has been supported by the French "ACI Masses de données" program, via the Geocomp project, and via the Brazilian Ministry of Science (MCT/CNPq 02/2006)

${ }^{\dagger}$ Corresponding author
}

Permission to make digital or hard copies of all or part of this work for personal or classroom use is granted without fee provided that copies are not made or distributed for profit or commercial advantage and that copies bear this notice and the full citation on the first page. To copy otherwise, to republish, to post on servers or to redistribute to lists, requires prior specific permission and/or a fee.

SCG'08, June 9-11, 2008, College Park, Maryland, USA.

Copyright 2008 ACM 978-1-60558-071-5/08/04 ...\$5.00. applications a new planarity criterion in terms of poset dimensions [31], as well as a very elegant and simple straightline drawing algorithm [32]. There are several equivalent formulations of Schnyder woods, either in terms of angle labeling (Schnyder labeling) or edge coloring and orientation or in terms of orientations with prescribed outdegrees. The most classical formulation is for the family of maximal plane graphs, i.e., plane triangulations, yielding the following striking property: the internal edges of a triangulation can be partitioned into three trees that span all inner vertices and are rooted respectively at each of the three vertices incident to the outer face. Schnyder woods, and more generally $\alpha$-orientations, received a great deal of attention [32, $17,22,19]$. From the combinatorial point of view, the set of Schnyder woods of a fixed triangulation has an interesting lattice structure $[7,3,18,13,14]$, and the nice characterization in terms of spanning trees motivated a large number of applications in several domains such as graph drawing [32, 22], graph coding and sampling $[12,21,4,29,20,5,10,1]$. Previous work focused mainly on the application and extension of the combinatorial properties of Schnyder woods to 3connected plane graphs $[17,22]$. In this article, we deal with combinatorial surfaces possibly having handles, i.e., oriented surfaces of arbitrary genus $g \geq 0$. Our main contribution is to show that it is possible to extend the local properties of Schnyder labeling in a coherent manner to triangulated surfaces. We investigate an important class of graphs, namely genus $g$ triangulations, corresponding to the combinatorial structure underlying manifold triangle meshes of genus $g$.

\subsection{Related Work}

Vertex spanning tree decompositions.

In the area of tree decompositions of graphs there exist some works dealing with the higher genus case. We mention one recent attempt to generalize Schnyder woods to the case of toroidal graphs [6] (genus 1 surfaces), based on a special planarizing procedure. In the genus 1 case it is actually possible to choose two adjacent non-contractible cycles, defining a so-called tambourine, whose removal makes the graph planar; the graph obtained can thus be endowed with a Schnyder wood. In the triangular case this approach yields a process for computing a partition of the edges into three edge-disjoint spanning trees plus at most 3 edges. Unfortunately, as pointed out by the authors, the local properties of Schnyder woods are possibly not satisfied for a large number of vertices, because the size of the tambourine might be arbitrary large. Moreover, it is not clear how to generalize the method to genus $g \geq 2$. 


\section{Planarizing surface graphs.}

A natural solution to deal with Schnyder woods (designed for planar graphs) in higher genus would consist in performing a planarization of the surface. Actually, given a surface $\mathcal{S}$ of genus $g$ and size $n$, one can compute a cut-graph or a collection of $2 g$ non-trivial cycles, whose removal makes $\mathcal{S}$ a topological disk (possibly with boundaries). There is a number of recent works [8, 15, 16, 24, 25, 35] dealing with interesting algorithmic challenges concerning the efficient computing of cut-graphs, optimal (canonical) polygonal schemas and shortest non-trivial cycles. For example some works make it possible to compute polygonal schemas in optimal $O(g n)$ time for a triangulated orientable manifold $[25,35]$. Nevertheless we point out that a strategy based on such planarizing approach would not be best suited for our purposes. From the combinatorial point of view this would imply to deal with boundaries of arbitrary size (arising from the planarizing procedure), as non-trivial cycles can be of size $\Omega(\sqrt{n})$, and cut-graphs have size $O(g n)$. Moreover, from the algorithmic complexity point of view, the most efficient procedures for computing small non-trivial cycles [8, 24] require more than linear time, the best known bound being currently of $O(n \log n)$ time.

\section{Schnyder trees and graph encoding.}

One of our main motivations for generalizing Schnyder woods to higher genus is the great number of existing works in the domain of graph encoding and mesh compression that take advantage of spanning tree decompositions [23, 30, 33], and in particular of the ones underlying Schnyder woods (and related extensions) for planar graphs $[1,11,12,20$, 21, 29]. The combinatorial properties of Schnyder woods and the related characterizations (canonical orderings [22]) for planar graphs yield efficient procedures for encoding tree structures based on multiple parenthesis words. In this context a number of works have been proposed for the simple compression [21] or the succinct encoding [12, 11] of several classes of planar graphs. More recently, this approach based on spanning tree decompositions has been further extended to design a new succinct encoding of labeled planar graphs [1]. Once again, the main ingredient is the definition of three traversal orders on the vertices of a triangulation, directly based on the properties of Schnyder woods. Finally we point out that the existence of minimal orientations (orientations without counter-clockwise directed cycles) recently made it possible to design the first optimal (linear time) encodings for some popular classes of planar graphs. For the case of triangulations and 3-connected plane graphs [20, 29 ] there exist some bijective correspondences between such graphs and some special classes of plane trees, which give nice combinatorial interpretations of enumerative formulas originally found by Tutte [34]. Very few works have been proposed for dealing with higher genus embedded graphs (corresponding to manifold meshes): this is due to the strong combinatorial properties involved in the planar case. Nevertheless, the topological approach used by Edgebreaker (using at most 3.67 bits per vertex in the planar case) has been successfully adapted to deal with triangulated surfaces having arbitrary topology: orientable manifold with handles [27] and also multiple boundaries [26]. Using a different approach, based on a partitioning scheme and a multi-level hierarchical representation [9], it is also possible to obtain an encoding of a triangulation of fixed genus $g$, having $f$ faces and $n$ vertices, requiring $2.175 f+O(g \log f)+o(f)$ bits (or $4.35 n+o(g n)$ bits) which is optimal for surfaces with a boundary: nevertheless, the amount of additional bits hidden in the sub-linear $o(n)$ term can be quite large, of order $\Theta\left(\frac{n}{\log n} \log \log n\right)$.

\subsection{Contributions}

Our first result consists in defining new traversal orders of the vertices of a triangulation of genus $g$, as an extension of the canonical orderings defined for planar graphs. We are also able to provide a generalization of the Schnyder labeling to the case of higher genus surfaces. The major novelty is to show that the linear time algorithm designed for the planar case can be extended in a nontrivial way in order to design a traversal of a genus $g$ surface. This induces a special edge coloring and orientation that is a natural generalization of the corresponding planar structure. In particular, the spanning property characterizing Schnyder wood is again verified almost everywhere in the genus $g$ case. Our approach involves the implicit computation of cut-graphs, as one would expect. Nevertheless, our strategy is really different from the one based on planarizing procedures: even if our cut-graphs and non-trivial cycles can have arbitrary size, we show that it is always possible to propagate the spanning condition of Schnyder woods even along this cuts in a coherent and natural manner. This property, and in particular the fact that there are only few exceptions to the spanning condition, allow us to characterize our graph decomposition in terms of one-face maps of genus $g$ (a natural generalization of plane trees). As application, we propose an encoding scheme allowing to represent the combinatorial information of a planar triangulation of size $n$ with at most $4 n+O(g \log n)$ bits. This is a natural extension of similar results obtained in the planar case $[21,2]$. Compared to previous existing works on graph encoding in higher genus, our result matches the same information theory bounds of the Edgebreaker scheme [30], which uses at most $3.67 n$ bits in the planar case, but requires up to $4 n+O(g \log n)$ bits for meshes with not spherical topology $[27,26]$.

\section{PRELIMINARIES}

\subsection{Graphs on surfaces.}

In this work we consider discrete surfaces such as triangular meshes, and we consider only the topological information specific to such a surface, i.e., the incidences vertices/edges/faces. All discrete surfaces we consider can be precisely formalized with the concept of map. A map is a graph embedded on a surface $\mathcal{S}$ such that the areas delimited by the graph, called the faces of the map, are topological disks. In the following the maps considered have neither loop nor multiple edges, which means that the embedded graphs are simple. Of particular interest for us are maps of genus $g$ with all faces of degree 3 ; we call such a map a triangulated surface of genus $g$. These maps represent the combinatorial incidences of triangular meshes of a surface of genus $g$. Given a surface $\mathcal{S}$, we refer to its primal graph (or 1-skeleton) as the underlying graph consisting of its edges and vertices. The dual graph of $\mathcal{S}$ is defined as the graph having the faces as nodes and whose edges correspond to pairs of adjacent faces in $\mathcal{S}$. Given a map $M$ on a surface $\mathcal{S}$, a cut graph of $M$ is a subgraph of $M$ whose induced embedding on $\mathcal{S}$ defines a one-face map. If $\mathcal{S}$ is cut along such a 


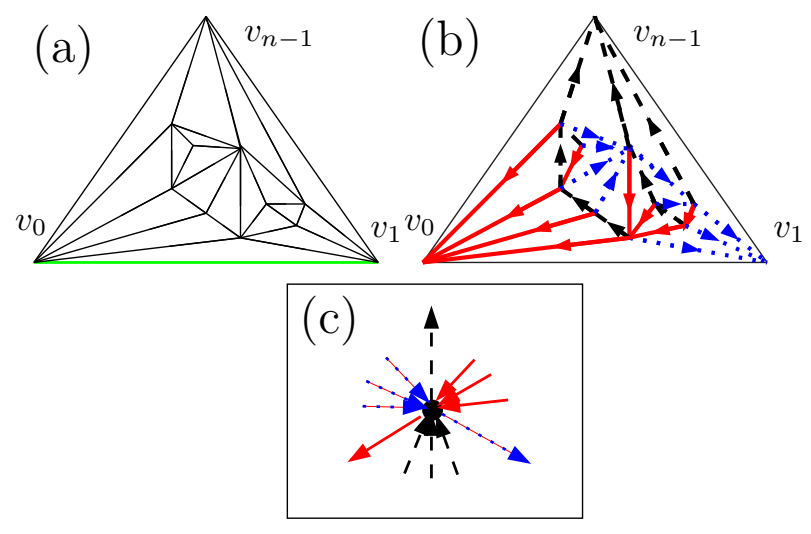

Figure 1: (a) A rooted planar triangulation, (b) endowed with a Schnyder wood. (c) The local condition of Schnyder woods.

submap $G^{c}$, the surface obtained is equivalent to a topological disk; the boundary of the disk contains each edge of $G^{c}$ twice, and the original surface can be obtained by identifying and gluing together these pairs of edges, which defines a so-called polygonal scheme of the surface $\mathcal{S}$ (for more details on the topological properties of graphs on surfaces see [28]).

\subsection{Schnyder woods for Plane Triangulations}

Let us first recall the definition of Schnyder woods for plane triangulations, which we will later generalize to higher genus.

While the definition is given in terms of local conditions, the main structural property is more global, namely a partition of the edges into 3 (essentially) spanning trees (see Figure 1).

Definition 1 ([32]). Let $T$ be a plane triangulation, and denote by $v_{0}, v_{1}, v_{n-1}$ the vertices of the outer face in cw order; we denote by $\mathcal{E}$ the set of edges of $T$ except those three incident to the outer face. A Schnyder wood of $\mathcal{T}$ is an orientation and labeling, with label in $\{0,1,2\}$ of the edges in $\mathcal{E}$ so as to satisfy the following conditions:

- root face condition: the edges incident to the vertices $v_{0}, v_{1}, v_{n-1}$ are all ingoing and are respectively of color 0,1 , and 2 .

- local condition: For each vertex $v$ not incident to the root face, the edges incident to $v$ in ccw order are: one outgoing edge colored 0 , zero or more incoming edges colored 2, one outgoing edge colored 1, zero or more incoming edges colored 0 , one outgoing edge colored 2 , and zero or more incoming edges colored 1, which we write concisely as

(Seq(In 1), Out 0, Seq(In 2), Out 1, Seq(In 0), Out 2).

A fundamental property concerning Schnyder woods [32] is expressed by the following:

FACT 1. Each plane triangulation $T$ admits a Schnyder wood. Given a Schnyder wood on $T$, the three oriented graphs $T_{0}, T_{1}, T_{2}$ induced by the edges of color $0,1,2$ are trees that span all inner vertices and are naturally rooted at $v_{0}, v_{1}$, and $v_{n-1}$, respectively.
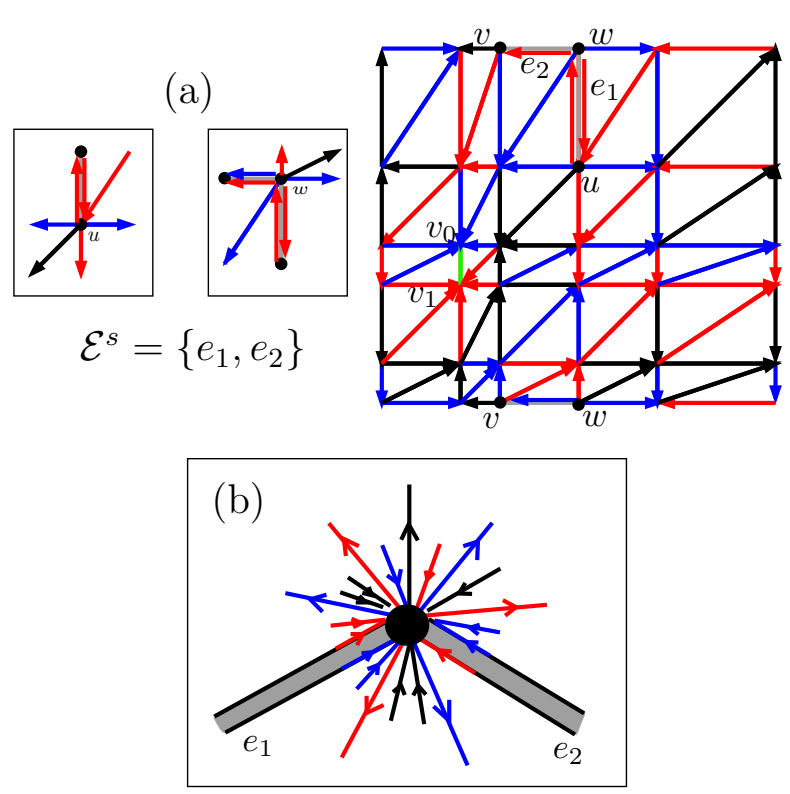

Figure 2: (a) A face-rooted triangulated surface $\mathcal{S}$ of genus 1 endowed with a $g$-Schnyder wood. (b) The local condition for vertices incident to special edges.

\section{HIGHER GENUS TRIANGULATIONS}

\subsection{Generalized Schnyder Woods}

As done in previous works considering the extension of Schnyder woods to other classes of (not triangulated) planar graphs [17, 29], one main contribution is to propose a new generalized version of Schnyder woods to genus $g$ triangulations (see Figure 2 for an example).

Definition 2. Consider a triangulated surface $\mathcal{S}$ of genus $g$, having $n$ vertices and a root face $\left(v_{0}, v_{1}, v_{n-1}\right)$; let $\mathcal{E}$ be the set of edges of $\mathcal{S}$ except those three incident to the root face. $A$ genus- $g$ Schnyder wood on the surface $\mathcal{S}$ is a partition of $\mathcal{E}$ into a set of normal edges and a set $\mathcal{E}^{s}=\left\{e_{1}, e_{2}, \ldots, e_{2 g}\right\}$ of $2 g$ special edges considered as fat, i.e., made of two parallel edges. In addition, each edge, a normal edge or one of the two edges of a special edge, is simply oriented and has a label in $\{0,1,2\}$, so as to satisfy the following conditions:

- root face condition: All edges incident to $v_{0}, v_{1}$, and $v_{n-1}$ are ingoing with color 0,1 , and 2 , respectively.

- local condition for vertices not incident to special edges: for every vertex $v \in \mathcal{S} \backslash\left(\left\{v_{0}, v_{1}, v_{n-1}\right\}\right.$ not incident to any special edge, the edges incident to $v$ in ccw order are, as in the planar case, of the form:

$$
\text { (Seq(In 1), Out 0, Seq(In 2), Out 1, Seq(In 0), Out 2). }
$$

- local condition for vertices incident to special edges: A vertex $v$ incident to $k \geq 1$ special edges has exactly one outgoing edge in color 2 . Consider the $k+1$ sectors around $v$ delimited by the $k$ special edges and the outgoing edge in color 2 . Then in each sector, the edges occur as follows in ccw order:

$$
\text { Seq(In 1), Out 0, Seq(In 2), Out 1, Seq(In 0). }
$$


The edge-based characterization of a triangulated surface proposed above has some advantages that make it a natural generalization of the planar definition. As one would expect, the planar and the genus $g$ definitions do coincide when considering planar triangulations. Second we observe that the local conditions hold at every vertex except the extremities of the $2 g$ edges in $\mathcal{E}^{s}$, where very similar conditions are satisfied. In particular almost all the vertices have out-degree 3, which makes the g-Schnyder woods a good characterization of the local planarity of a bounded genus surface. Finally, we point out that the local condition above leads to graph decompositions expressed in terms of one-face maps having genus $g$, which are the natural generalization of plane spanning trees (see Theorem 8 and the remark after).

\subsection{Handle operators}

Following the approach suggested in [27], based on Handlebody theory for surfaces, we design new traversal strategy for higher genus surfaces: as in the planar case, our strategy consists in conquering the whole graph incrementally, face by face, using a vertex-based operator (conquer) and two new operators (split and merge) designed to represent the handle attachments. We start by setting some notations and definitions. We consider a triangulated surfaces $\mathcal{S}$ having genus $g$ and $n$ vertices. We denote by $S^{\text {in }}\left(S^{\text {out }}\right)$ the embedded growing subgraph of $\mathcal{S}$ induced by the faces already conquered (not yet conquered, respectively). $S^{\text {out }}$ is a face-connected map of genus $g$ having $b \geq 1$ boundaries, each boundary being a simple cycle $C_{i}, i \in[1 . . b]$. We define $\partial S^{i n}:=\cup_{i=1}^{b} C_{i}$ as the overall border between $S^{\text {in }}$ and $S^{\text {out }}$.

\section{Handle operator of first type.}

Definition 3. A chordal edge is an edge of $S^{\text {out }} \backslash \partial S^{\text {in }}$ whose two extremities are on $\partial S^{i n}$. A boundary vertex $w \in$ $C_{i}$ is free if $w$ is not incident to a chordal edge $e$.

We can now introduce the first operator, called conquer, which adds a free vertex $w$ with all its incident edges and faces to $S^{i n}$. More precisely, the conquest or removal of a vertex $w$ consists in attaching a set of triangles to $S^{i n}$ as follows (see the first picture in Figure 3):

- conquer $(w)$, with $w$ a free vertex: Update the conquered area, by transferring from $S^{\text {out }}$ to $S^{\text {in }}$ all faces incident to $w$ that were not yet in $S^{\text {in }}$. The vertices and edges of $S^{\text {out }}$ $\left(S^{i n}\right)$ are naturally updated as those vertices and edges incident to the faces of $S^{\text {out }}$ ( $S^{\text {in }}$, respectively).

This operation corresponds to a handle operator of type 1 (see [27]), not modifying the topology of $S^{i n}$.

\section{Handle operators of second type.}

Consider a triangulated surface $\mathcal{S}$ of genus $g$ and a collection of $b$ cycles $\left\{C_{i}\right\}_{i}$ delimiting a face-connected map $S^{\text {out }}$. A chordal edge $e$ for $S^{\text {out }}$ is said to be non-separating if $S^{\text {out }}$ is not disconnected when cutting along $e$. A chordal edge whose two extremities are in the same cycle $C_{i}$ is called a splitting chordal edge. For such an edge, we define $C^{\prime}$ and $C^{\prime \prime}$ as the two cycles formed by $C_{i}+e$. Then $e$ is said to be contractible if either $C^{\prime}$ or $C^{\prime \prime}$ is contractible. Note that a non-separating splitting chordal edge can not be contractible.

Definition 4 (SPlit EDGE). A split edge for the area $S^{\text {out }}$ is a non-separating splitting chordal edge.
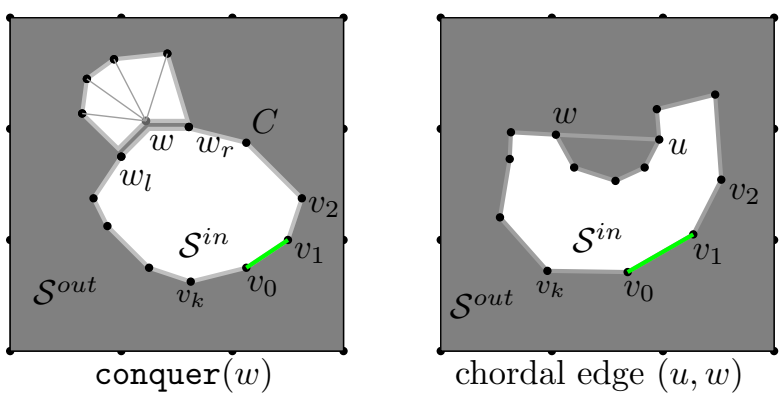

chordal edge $(u, w)$
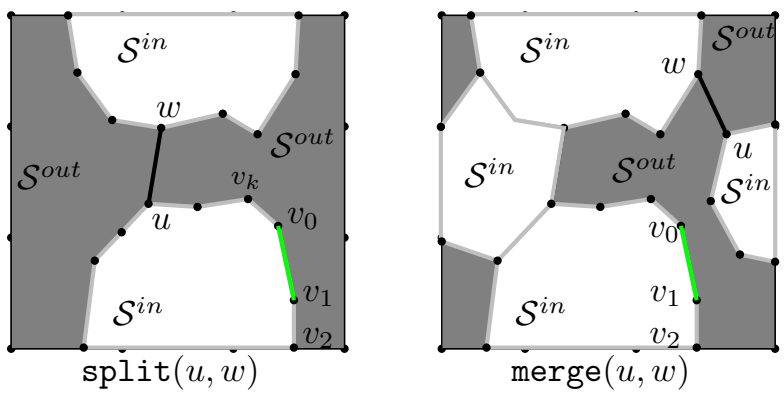

Figure 3: Illustrated on a toroidal graph, from left to right, a conquer operation, a contractible chordal edge, a split edge, and a merge edge.

It is easy to see that the removal of a split edge $e$ produces a face-connected map $S^{\text {out }} \backslash\{e\}$ with $b+1$ boundaries, hence having Euler characteristic $\chi^{\prime}=2-2 g-(b+1)=\chi-1$.

Definition 5 (MERGe EDGE). A merge edge for the area $S^{\text {out }}$ is a non-separating chordal edge e having its two extremities on two distinct cycles $C_{i}$ and $C_{j}, i \neq j$.

This time the removal of the edge $e$ produces a faceconnected map $S^{\text {out }} \backslash e$ with $b-1$ boundaries, hence having Euler characteristic $\chi^{\prime}=2-2 g-(b-1)=\chi+1$.

We are now able to define two new operators to perform handle attachments. The split and merge operators defined below are (the inverse of) handle operators of type 1: they are designed to identify boundary vertices lying on distinct cycles. Intuitively, the split operator splits a boundary curve into two distinct components, thereby increasing the number of boundaries of $\partial S^{i n}$ by 1 ; while a merge operator merges two boundaries, thereby decreasing the number of boundaries of $\partial S^{i n}$ by 1 .

Let $\mathcal{S}$ be a triangulated surface of genus $g$ and let $C_{i}, i \in$ [1..b] be a collection of $b$ cycles delimiting a face-connected map $S^{\text {out }}$. Given a split edge $e=(u, w)$ having its extremities on a cycle $C$, the operator split $(e)$ produces the splitting of $C=\left\{v_{0}, v_{1}, \ldots, v_{k}\right\}$ (with $u=v_{i_{1}}$ and $w=v_{i_{2}}$, for some indices $\left.i_{1}<i_{2} \leq k\right)$ into two new cycles $C^{\prime}, C^{\prime \prime}$. More precisely $C^{\prime}$ is the new simple cycle defined by the set of vertices $\left\{v_{0}, v_{1}, \ldots, v_{i_{1}}^{\prime}, v_{i_{2}}^{\prime}, v_{i_{2}+1}, \ldots, v_{k-1}, v_{k}\right\}$, where $v_{i_{1}}^{\prime}$ and $v_{i_{2}}^{\prime}$ are copies of the vertices $v_{1}$ and $v_{2}$. In similar way, a new cycle $C^{\prime \prime}$ is created by considering two duplicated vertices $v_{i_{1}}^{\prime \prime}$ and $v_{i_{2}}^{\prime \prime}$ (copies of $v_{i_{1}}$ and $v_{i_{2}}$ ) and the list of vertices $\left\{v_{i_{1}}^{\prime \prime}, v_{i_{2}}^{\prime \prime}, v_{i_{2}}+1, \ldots, v_{i_{1}}-1\right\}$.

A merge operation can be performed in a similar way, on a given merge edge $e=\left(v_{i_{1}}^{\prime}, v_{i_{2}}^{\prime \prime}\right)$ whose extremities belong to different boundaries $C^{\prime}$ and $C^{\prime \prime}$, and produces a new simple cycle $\bar{C}$ containing two copies of $v_{i_{1}}^{\prime}$ and $v_{i_{2}}^{\prime \prime}$ and all the vertices in $C^{\prime}$ and $C^{\prime \prime}$. More precisely, denoting by 

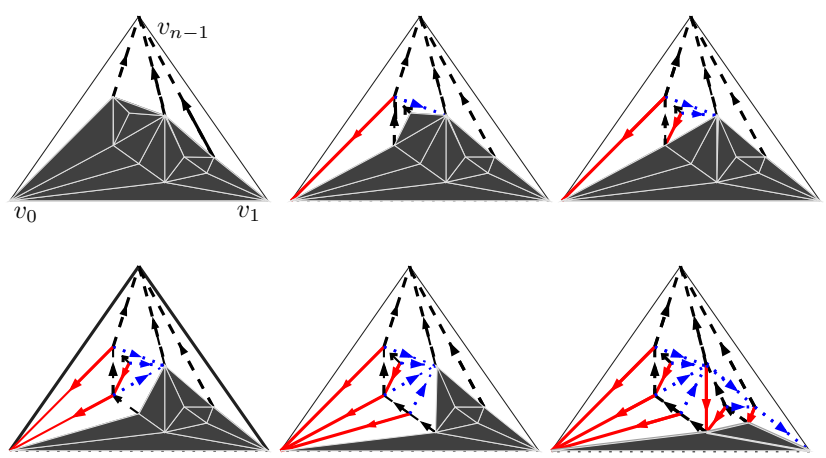

Figure 4: Some steps of the algorithm that computes a Schnyder wood of a planar triangulation $\mathcal{T}$ (rooted at $\left.\left(v_{0}, v_{1}\right)\right)$. The traversal is performed as a sequence of conquer operations on free vertices: edges in $S^{\text {out }}$ (dark region) are oriented and directed according to the Schnyder rule.

$\left\{v_{0}^{\prime}, \ldots, v_{k^{\prime}}^{\prime}\right\}$ the set of vertices defining $C^{\prime}$ and $\left\{v_{0}^{\prime \prime}, \ldots, v_{k^{\prime \prime}}^{\prime \prime}\right\}$ the vertices of $C^{\prime \prime}$, the new simple cycle $\bar{C}$ is defined by $\left\{v_{0}^{\prime}, \ldots, v_{i_{2}}^{\prime}, v_{i_{2}}^{\prime \prime}, v_{i_{2}+1}^{\prime \prime}, \ldots, v_{k^{\prime \prime}}^{\prime \prime}, v_{0}^{\prime}, \ldots, v_{i_{2}}^{\prime \prime}, v_{i_{1}}^{\prime}, v_{i_{1}+1}^{\prime}, \ldots, v_{k^{\prime}}^{\prime}\right\}$

The edges involved in the merge/split operations are respectively called merge edges and split edges. These will be exactly the special edges of the genus $g$ Schnyder wood to be computed by our traversal algorithm The vertices incident to merge or split edges are called multiple vertices, as each merge/split operation results in considering such a vertex as duplicated.

\subsection{Computing a Schnyder wood of a planar triangulation}

In this section we briefly review a well-known linear time algorithm designed for computing a Schnyder wood of a planar triangulation, following the presentation by Brehm [7]. Once defined the conquer operation, we can associate to it a simple rule for coloring and orienting the edges incident to a vertex conquered:

- colorient $(v)$ : orient outward of $v$ the two edges connecting $v$ to its two neighbors on $\partial S^{\text {in }}$; assign color 0 (1) to the edge connected to the right (left, respectively) neighbor, looking toward $S^{\text {out }}$. Orient toward $v$ and color 2 all edges incident to $v$ in $S^{\text {out }} \backslash \partial S^{\text {in }}$.

We can now formulate the algorithm for computing a Schnyder wood as a sequence of $n-2$ conquer and colorient operations, following a simple and elegant presentation adopted by Brehm [7]. Given a plane triangulation $\mathcal{T}$ with outer face $\left\{v_{0}, v_{1}, v_{n-1}\right\}$ we start with $S^{i n}$ - the set of visited vertices - initialized to $\left\{v_{0}, v_{1}, v_{n-1}\right\}$, and with $\partial S^{i n}$ - the border of $S^{i n}$ — initialized to be the contour of the outer face.

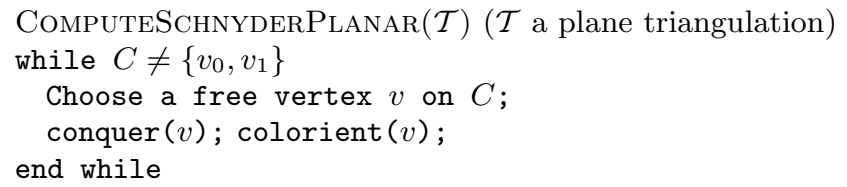

The correctness and termination of the traversal algorithm above is based on the fundamental property that a triangulated topological disk always has on its boundary a vertex not incident to any internal chord.

One major advantage of computing a Schnyder wood in an incremental way is that we are able to put in evidence some invariants, which remain satisfied at each step of the algorithm:

- the edges that are already colored and oriented are those in $S^{i n} \backslash \partial S^{i n}$;

- for each vertex $v \in S^{i n} \backslash \partial S^{i n}$, all edges incident to $v$ are colored and directed in such a way that the Schnyder rule is always satisfied;

- every boundary node $v \in C \backslash\left\{v_{0}, v_{1}\right\}$ has exactly one outgoing edge lying in $S^{i n} \backslash \partial S^{i n}$. This edge $e$ has color 2 , and the ingoing edges in $S^{i n} \backslash \partial S^{i n}$ incident to $v$ between $e$ and the right (left) neighbour of $v$, looking toward $S^{\text {out }}$, have color 1 (0, resp.).

Using these invariants, it is straightforward to prove the following lemma (see [7] for a detailed presentation):

LEMma 6. Given a planar triangulation $\mathcal{T}$ with outer face $\left(v_{0}, v_{1}, v_{n-1}\right)$ the algorithm COMPUTESCHNYDERPLANAR computes in linear time a Schnyder wood of $\mathcal{T}$.

\subsection{A new traversal for genus $g$ surfaces}

This section presents our main contribution, an algorithm for traversing a triangulated surface $\mathcal{S}$ of arbitrary genus $g \geq 0$, which extends the algorithm of Brehm in a natural way. As in the planar case, the traversal is a greedy sequence of conquer operations on free vertices, with here the important difference that these operations are interleaved with $2 g$ merge/split operations so as to make the genus of the conquered area increase from 0 to $g$. We start with $\mathcal{C}:=\left\{C_{0}\right\}:=\left\{\left(v_{0}, v_{1}, v_{n-1}\right)\right\}$. At the beginning $S^{i n}$ is a topological disk delimited by the simple cycle $C_{0}$. As in the planar case, we make use of the operation conquer $(v)$ that consists in transferring the (remaining) faces incident to $v$ from $S^{\text {out }}$ to $S^{\text {in }}$. Associated with such a conquest is the colorient rule for coloring and orienting the edges that are conquered, as defined in Section 3.3. We also make use of the handle operations split and merge, as defined in Section 3.2 .

ComputeSchnyderAnyGenus $(\mathcal{S})$ ( $\mathcal{S}$ a triangulated surface of genus $g$ )

while $\{\mathcal{C}\} \neq\left\{v_{0}, v_{1}\right\}$

If there exists a free vertex $v$ on some $C_{i} \in \mathcal{C}$ conquer $(v)$; colorient $(v)$;

otherwise, if there exists a split edge $e=(u, w) \in$ $S^{\text {out }} \backslash \partial S^{\text {in }}$;

split $(\mathrm{u}, \mathrm{w})$; thereby adding a new cycle $C^{\prime}$ to $\mathcal{C}$;

otherwise, find a merge edge $e=(u, w) \in S^{o u t}$; merge $(u, w)$; thereby removing a cycle from $\mathcal{C}$; end while

Figure 7 shows the traversal algorithm executed on a toroidal triangulation.

Notice the subtlety that, for nonzero genus, the multiple vertices, i.e., the vertices incident to merge/split edges, are conquered several times, as illustrated in Figure 6. Precisely, for a vertex $v$ incident to $k \geq 0$ merge/split edges, conquer $(v)$ occurs $k+1$ times.

Lemma 7. For any triangulated surface $\mathcal{S}$ of genus $g$, ComputeSchnyderAnyGenus $(\mathcal{S})$ terminates, and can be implemented to run in linear time. 
(planar case)

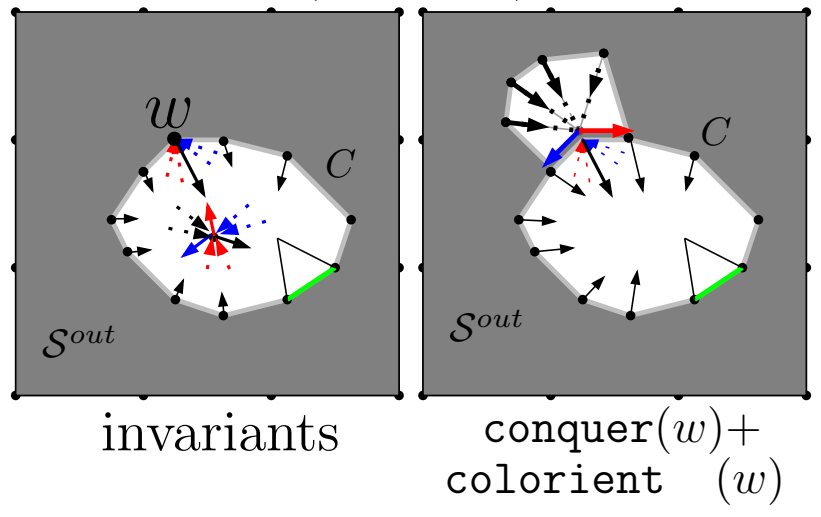

Figure 5: These pictures show the result of a colorient operation in the planar case.

(toroidal case)
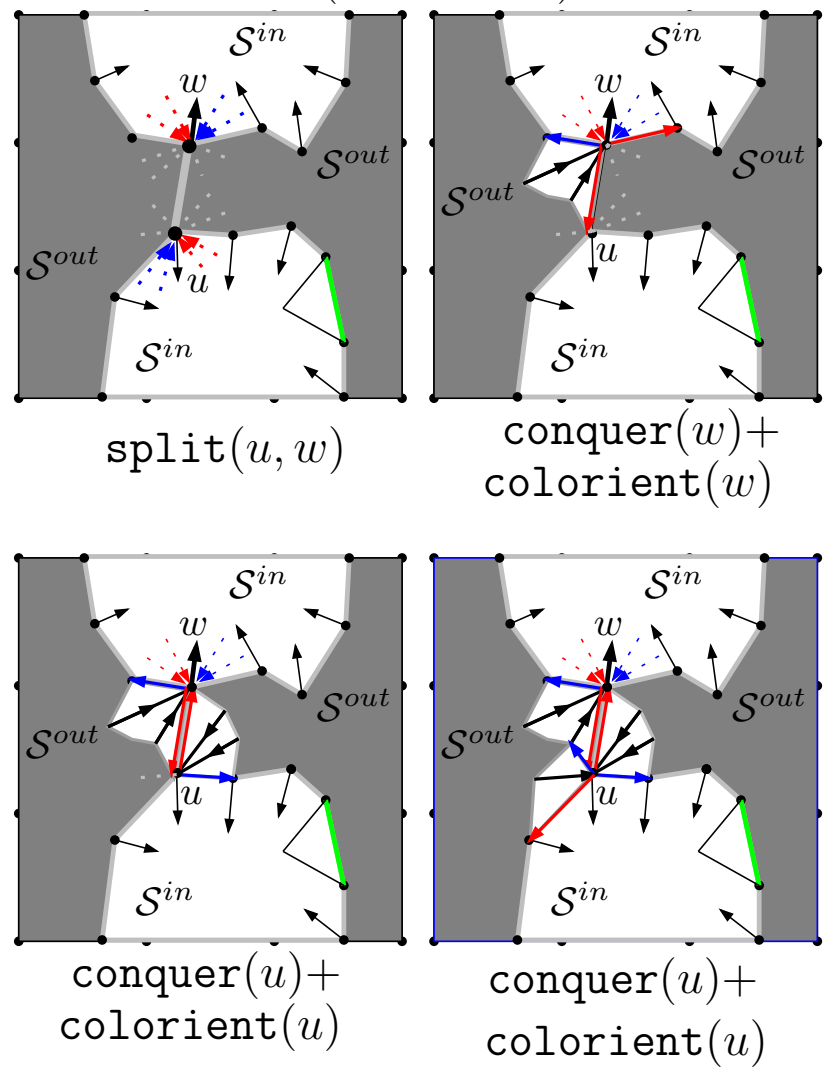

Figure 6: These pictures show the result of colorient operations in the higher genus case. Any chordal split or merge edge can be oriented in one or two directions (having possible two colors), depending on the traversal order on its extremities.
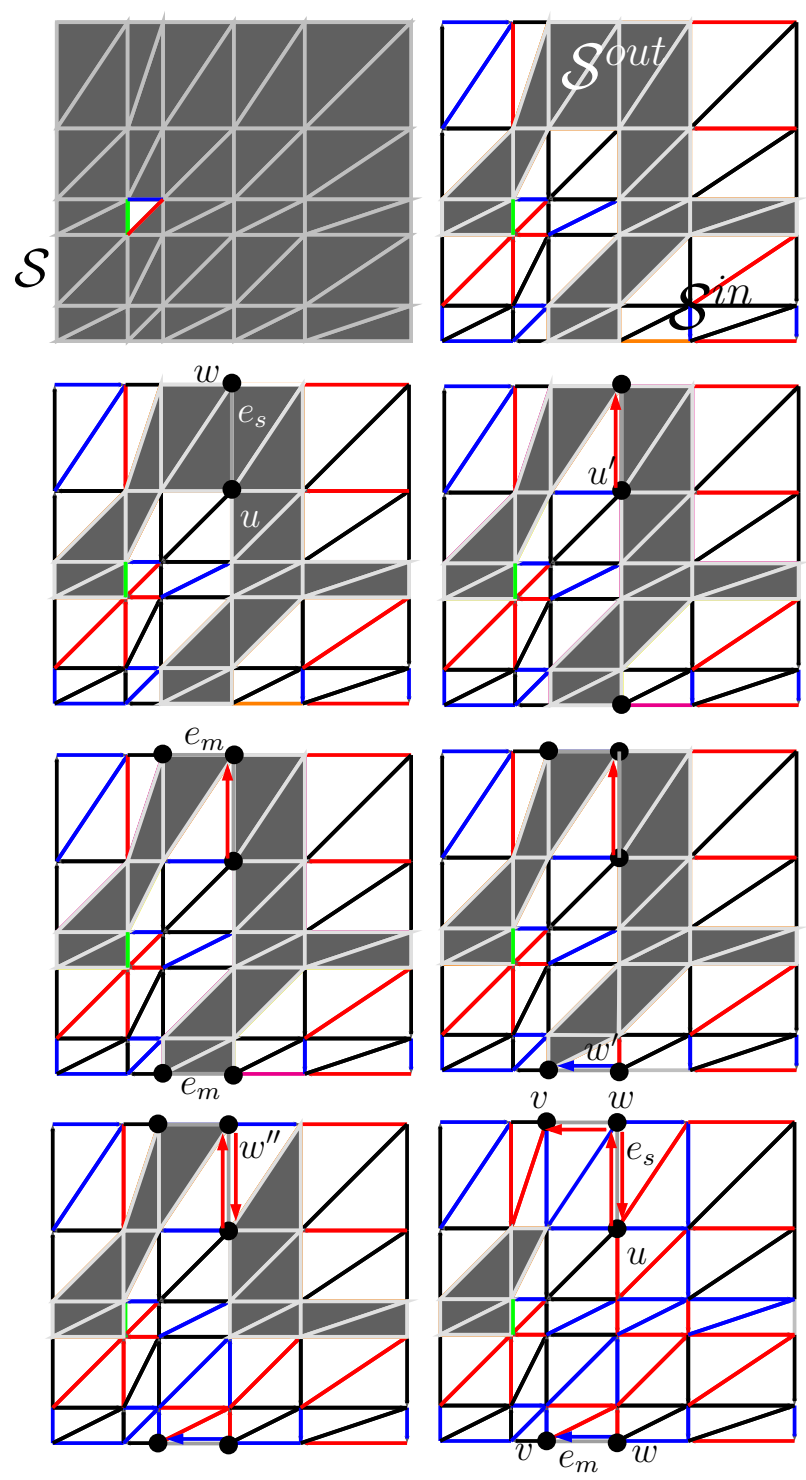

Figure 7: An example of execution of our traversal algorithm for a triangulated surface of genus 1. As in the planar case the traversal starts from the root face (the one incident to the green root edge). As far as only conquer operations on free vertices are performed, the area already explored (white triangles) remains planar and homeomorphic to a disk. When there remain no free vertices to conquer (second Figure), it is possible to find split and merge edges (incident to black circles). After performing split and merge operations, (here on the edges $(u, w)$ and $(w, v))$, new free vertices can be found (here $u^{\prime}$, $w^{\prime}$, and $\left.w^{\prime \prime}\right)$ in order to continue the traversal, until the entire graph is visited. 
Proof. (elements) To prove that the traversal terminates, it suffices to show that a merge or split operation is always possible whenever there remain no free vertices on $\partial S^{i n}$. To prove this, we use the property that a contractible chord for $\partial S^{i n}$ yields a free vertex on $\partial S^{i n}$ (precisely, a free vertex in the subpath $P$ of $\partial S^{i n}$ such that $P+e$ is a contractible cycle). Hence, if there remain no free vertices, then each vertex on $\partial S^{i n}$ is incident to a chordal edge, and all chordal edges are non-contractible. In that case, let $v$ be a vertex on $\partial S^{i n}$ and let $e$ be a chordal edge incident to $v$. If $e$ is non-separating, then we are fine. Otherwise, cutting $S^{\text {out }}$ along $e$ splits $S^{\text {out }}$ into two smaller surfaces $S_{1}$ and $S_{2}$. By an inductive argument, there exists a non-separating chordal edge for $\partial S_{1}$, so we are done.

Implementing the algorithm to run in linear time requires some bookkeeping. For this purpose, we maintain the list of chordal edges for $\partial S^{i n}$, and, which is more difficult, we maintain a classification of these chordal edges as contractible, non-contractible separating, split edges, and merge edge. Such a classification is done by maintaining a spanning cutgraph $G^{c}$ for the dual of $S^{\text {out }}$, such that $G^{c}$ contains the dual of all chordal edges; and by maintaining a depth-first-search spanning tree of $G^{c}$ so as to test which edges of $G^{c}$ disconnect $G^{c}$. All this bookkeeping can be done in amortized linear cost.

ThEOREM 8. Let $\mathcal{S}$ be a triangulated surface of genus $g$. Then the coloration and orientation of edges obtained at the end of the procedure COMPUTESCHNyderAnyGEnus $(\mathcal{S})$ is a genus $g$ Schnyder wood on $\mathcal{S}$. The Schnyder wood thus obtained has the additional property that the graph $T_{2}$ formed by the edges with color 2 is a tree, and the embedded graph formed by $T_{2}$ and the $2 g$ special edges is a one-face map; moreover the embedded graphs $T_{0}, T_{1}$ formed respectively by the edges of color 0 and of color 1 are genus $g$ maps with at most $1+2 g$ faces.

Proof. (elements) To prove that the combinatorial structure obtained by the traversal algorithm is a genus $g$ Schnyder wood, it suffices to define some invariants that remain satisfied all along the traversal. We make use of the invariants already defined for the planar case, as well as new invariants relative to multiple vertices, which state that the local condition is satisfied for multiple vertices in $S^{i n} \backslash \partial S^{i n}$, and is already partially satisfied for those on $\partial S^{i n}$.

Let us now sketch the proof of the second part of the theorem, i.e., the additional properties of $T_{0}, T_{1}$, and $T_{2}$. Observe that, by design of the traversal algorithm, $T_{2}$ is a connected (embedded) graph spanning all vertices of $\mathcal{S} \backslash\left\{v_{0}, v_{1}\right\}$. Moreover, all these vertices have out-degree 1 in $T_{2}$, except the root vertex $v_{n-1}$ that has out-degree 0 . Hence, $T_{2}$ is a tree spanning the vertices of $\mathcal{S} \backslash\left\{v_{0}, v_{1}\right\}$ and rooted at $v_{n-1}$. Moreover, the addition of each of the $2 g$ special edges creates a new fundamental cycle of the surface, so the embedded graph formed by $T_{2}$ and the $2 g$ special edges is a one-face map.

Concerning the edges of color $i \in\{0,1\}$, an argument based on some topological invariants ensures that all the faces of the sets $T_{i}$ are topological disks (hence defining a map). More precisely, by construction, a conquer operation does not change the topology of $T_{i}$, while a split or merge operation correspond to identify nodes in the three, which creates (by definition) new non-trivial cycles. Finally, the bound $1+2 g$ on the number of faces is due to the fact that each special edge contributes at most by one to the number of new faces of $T_{i}$.

REMARK 9. The properties of $T_{0}, T_{1}, T_{2}$ stated in Theorem 8 can be considered as extensions of the fundamental property of planar Schnyder woods, which states that $T_{0}$, $T_{1}$, and $T_{2}$ are plane trees. Figure 8 shows an example in genus 1 .
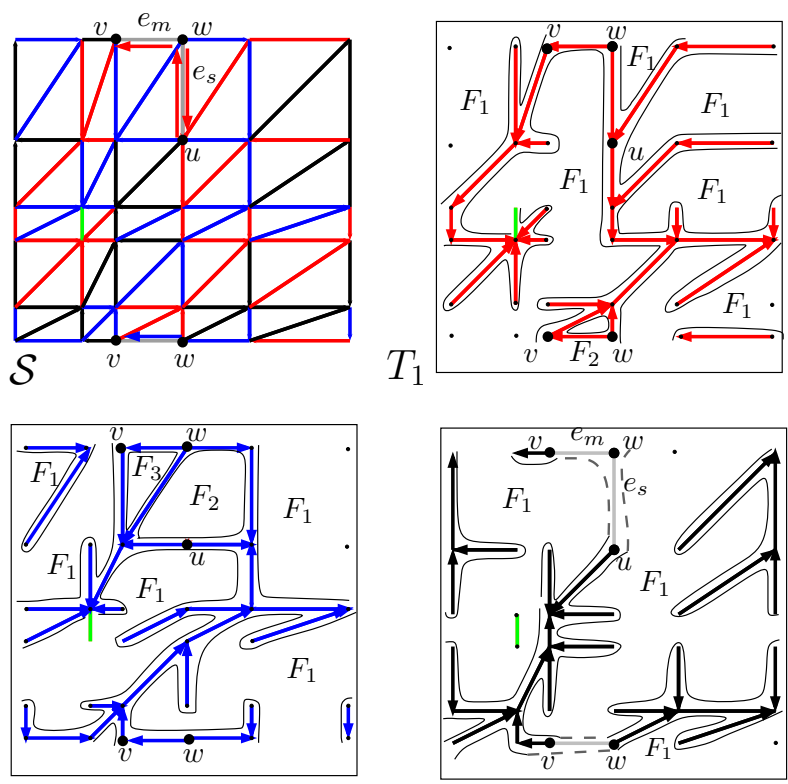

$T_{0}$

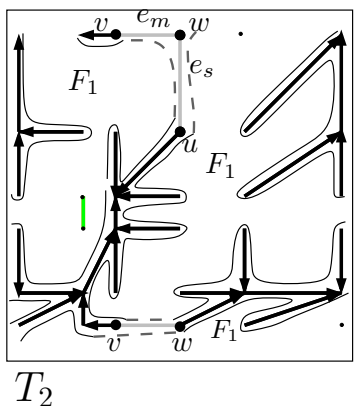

Figure 8: A triangulated torus endowed with a Schnyder wood. The black edges form a tree $T_{2}$, and the addition of the two special edges yields a one-face map. The embedded graph $T_{0}$ (blue edges) is a map with 3 faces; and the embedded graph $T_{1}$ (red edges) is a map with 2 faces.

\section{APPLICATION TO ENCODING}

In the planar case, Schnyder woods yield a simple encoding procedure for triangulations, as described in [21] and more recently in [2]. Precisely, a Schnyder wood with $n$ inner vertices is encoded by two parenthesis words $W, W^{\prime}$ each of size $2 n$, where $W$ is the classical Dyck encoding for the tree $T_{2}$; and $W^{\prime}$ is obtained from a clockwise walk around $T_{2}$ starting at $v_{n-1}$, writing an opening (closing) parenthesis each time an outgoing edge in color 1 (ingoing edge in color 0, resp.) is crossed, and completing the end of the word with closing parenthesis. Hence, for a triangulation with $n+3$ vertices, the coding word has length $4 n$. This code is both simple and quite compact, as the length $4 n$ is not far from the information-theory lower bound of $\left(\log _{2}\left(4^{4} / 3^{3}\right) \cdot n\right) \approx 3.245 \cdot n$, which is attained in the planar case by a bijective construction due to Poulalhon and Schaeffer [29].

Such a bijective construction is still to be found for higher genus, but we can here at least extend to higher genus the simple encoding procedure based on Schnyder woods. To encode the Schnyder wood we proceed in a similar way as in the planar case except that we have to deal with the 
special edges. First, the one-face map $M$ formed by $T_{2}$ and the special edges is encoded by the Dyck word $W$ for $T_{2}$, augmented by $O(g)$ pointers (each of size $O(\log (n))$ ) so as to locate the two extremities of each special edge. We obtain the second encoding word $W^{\prime}$ from a clockwise walk along the unique face of $M$ starting at $v_{n-1}$, writing an opening (closing) parenthesis each time an outgoing edge in color 1 (ingoing edge in color 0, resp.) is crossed, and completing the end of the word with closing parenthesis.

All in all, we obtain the following result:

Proposition 10. A triangulated surface of genus $g$ with $n+3$ vertices can be encoded in linear time -via a genus $g$ Schnyder wood - by two parenthesis words respectively of lengths $2 n$ and $2(n+4 g)$, plus $O(g)$ pointers each of length $O(\log (n))$; so the total code length is $4 n+O(g \log (n))$. Coding and decoding can be done in linear time.

We can also design a more sophisticated code that supports queries: this time we need to encode the three maps $T_{i}$ using multiple parenthesis systems (as done in $[12,1]$ ).

Given a (unlabelled) triangulation $\mathcal{S}$ of genus $g$ with $e$ edges, it is possible to obtain a compact representation of $\mathcal{S}$ using asymptotically $\left(2 \log _{2} 6\right) \cdot e+O(g \log e)$ bits which answers queries for vertex adjacency and vertex degree in $O(1)$ time. Once again the main idea is to compute a $\mathrm{g}$ Schnyder wood of $\mathcal{S}$ and to encode the corresponding maps $T_{i}, i \in\{0,1,2\}$. This time, in order to efficiently support adjacency queries on vertices we have to encode the three maps $T_{i}$ using several types of parentheses: we use a multiple parenthesis system (with 2 types of parentheses) as above to describe each map $T_{i}$ (the arguments are similar to the ones given in [1], where the planar labeled triangulations are considered) We mention that the partitioning strategy presented in [9] achieves a better bound of 2.175 bits per face, more $O(g \log n)$ additional bits for dealing with handles (with different face based navigation). Nevertheless, our approach could be successful in extending existing encodings for more general planar graphs ([12]) and for labeled graphs ([1]) to the higher genus case.

\section{CONCLUSION AND PERSPECTIVES}

We have presented a general approach for extending to higher genus a fundamental combinatorial structure, Schnyder woods, which is by now a standard tool to handle planar graphs both structurally and algorithmically. We have been successful in showing that the definition and several fundamental combinatorial properties can be extended from the planar to the genus $g$ case in a natural way. We think that this work is a first step in the attempt of generalizing the properties of Schnyder woods and more generally $\alpha$-orientations (orientations with prescribed out-degrees) to other interesting classes of graphs.

\section{Canonical orderings and Schnyder Woods.}

We point out that our graph traversal procedure induces an ordering for treating the vertices so as to shell the surface progressively. Such an ordering is already well known in the planar case under the name of canonical ordering and has numerous applications for graph encoding and graph drawing $[12,22]$. It is thus of interest to extend this concept to higher genus. The only difference is that in the genus $g$ case there is a small number - at most $2 \cdot 2 g$ - of vertices that might appear several times in the ordering; these correspond to the vertices incident to the $2 g$ special edges (split/merge edges) obtained during the traversal. There are several open questions we think should be investigated concerning the combinatorial properties of such orderings and the corresponding edges orientations and colorations. For example, one may ask whether to any edge coloration and coloring defining a Schnyder wood could correspond a canonical ordering, as in the planar case. We think also it would be interesting to see whether such an ordering would yield an efficient algorithm for drawing a graph on genus $g$ surface (as it has been done in the planar case [22]).

\section{Further extensions.}

Our approach relies on quite general topological and combinatorial arguments, so the natural next step should be to apply our methodology to other interesting classes of graphs (not strictly triangulated), which have similar characterization in the planar case. Our topological traversal could be extended to the 3-connected case, precisely to embedded 3connected graphs with face-width larger than 2, which correspond to polygonal meshes of genus $g$. We point out that our encoding proposed in Section 4 could take advantage of the existing compact encodings of planar graphs [12, 11, 21], using similar parenthesis-based approaches.

\section{Lattice structure.}

There are a number of deep and interesting questions that our work leaves open. From the combinatorial point of view it should be of interest to investigate whether edge orientations and colorations in genus $g$ have nice lattice properties, as in the planar case. In particular, the existence of minimal orientations in the planar case led to a large number of deep results in areas such as graph drawing, optimal sampling and coding.

\section{Graph encoding applications.}

In addition to the new tree decomposition proposed for the higher genus case, one contribution has been to show, from the algorithmic point of view, that vertex-decomposition based traversal can be performed in linear time for any genus $g \geq 0$.

In particular, the linear time complexity of our traversal procedure is due to the very low complexity, $O(1)$ time, to check that a chordal edge is non-contractible Thus a "leftmost" traversal of the triangulated surface should be doable in linear time (let us recall that a left-most driven traversal returns in the planar case the minimal orientations mentioned above). This remark, together with the local properties of genus $g$ Schnyder woods (Definition 2), suggests the possibility of further nice applications in graph encoding and sampling (see [20, 29] for the planar case).

More precisely, we conjecture the existence of correspondences between some special classes of one-face maps (generalizations of plane trees) and the classes of triangulated and polyhedral combinatorial meshes of genus $g$ : this would lead to the first (linear time) optimal compression schemes and succinct encodings for these fundamental classes of meshes.

\section{Acknowledgments}

We are grateful to N. Bonichon, C. Gavoille and A. Labourel for enlightening discussions on Schnyder woods. First author would like to thank E. Colin de Verdière for pointing out some useful topological properties of graphs on surfaces. 
We are extremely grateful to O. Bernardi and G. Schaeffer for enlightening discussions on the combinatorics of maps that motivated this work.

\section{REFERENCES}

[1] J. Barbay, L. Castelli-Aleardi, M. He, and J. I. Munro. Succinct representation of labeled graphs. In ISAAC, pages 316-328, 2007.

[2] O. Bernardi and N. Bonichon. Catalans intervals and realizers of triangulations. In Proc. FPSAC07, 2007.

[3] N. Bonichon. Aspects algorithmiques et combinatoires des réaliseurs des graphes plans maximaux. $\mathrm{PhD}$ thesis, Bordeaux I, 2002.

[4] N. Bonichon, C. Gavoille, and N. Hanusse. An information-theoretic upper bound of planar graphs using triangulation. In $S T A C S$, pages 499-510. Springer, 2003.

[5] N. Bonichon, C. Gavoille, N. Hanusse, D. Poulalhon, and G. Schaeffer. Planar graphs, via well-orderly maps and trees. Graphs and Combinatorics, 22(2):185-202, 2006.

[6] N. Bonichon, C. Gavoille, and A. Labourel. Edge partition of toroidal graphs into forests in linear time. In ICGT, volume 22, pages 421-425, 2005.

[7] E. Brehm. 3-orientations and schnyder-trhree tree decompositions. Master's thesis, Freie Universitaet Berlin, 2000.

[8] S. Cabello and B. Mohar. Finding shortest non-separating and non-contractible cycles for topologically embedded graphs. Discrete $\&$ Comp. Geometry, 37(2):213-235, 2007.

[9] L. Castelli-Aleardi, O. Devillers, and G. Schaeffer. Succinct representation of triangulations with a boundary. In WADS, pages 134-145. Springer, 2005.

[10] L. Castelli-Aleardi, O. Devillers, and G. Schaeffer. Optimal succinct representations of planar maps. In SoCG, pages 309-318, 2006.

[11] Y.-T. Chiang, C.-C. Lin, and H.-I. Lu. Orderly spanning trees with applications to graph encoding and graph drawing. In SODA, pages 506-515, 2001.

[12] R. C.-N. Chuang, A. Garg, X. He, M.-Y. Kao, and H.-I. Lu. Compact encodings of planar graphs via canonical orderings and multiple parentheses. ICALP, pages 118-129, 1998.

[13] H. de Fraysseix and P. O. de Mendez. On topological aspects of orientations. Disc. Math., 229:57-72, 2001.

[14] P. O. de Mendez. Orientations bipolaires. PhD thesis, Paris, 1994

[15] É. C. de Verdière and F. Lazarus. Optimal system of loops on an orientable surface. In FOCS, pages 627-636, 2002.

[16] J. Erickson and S. Har-Peled. Optimally cutting a surface into a disk. Discrete \& Comp. Geometry, 31(1):37-59, 2004.
[17] S. Felsner. Convex drawings of planar graphs and the order dimension of 3-polytopes. Order, 18:19-37, 2001.

[18] S. Felsner. Lattice structures from planar graphs. Electronic Journal of Combinatorics, 11(15):24, 2004.

[19] E. Fusy. Combinatoire des cartes planaires et applications algorithmiques. $\mathrm{PhD}$ thesis, Ecole Polytechnique, 2007.

[20] E. Fusy, D. Poulalhon, and G. Schaeffer. Dissections and trees, with applications to optimal mesh encoding and to random sampling. In SODA, pages 690-699, 2005.

[21] X. He, M.-Y. Kao, and H.-I. Lu. Linear-time succint encodings of planar graphs via canonical orderings. Journal on Discrete Mathematics, 12:317-325, 1999.

[22] G. Kant. Drawing planar graphs using the canonical ordering. Algorithmica, 16(1):4-32, 1996.

[23] K. Keeler and J. Westbrook. Short encondings of planar graph and maps. Discrete and Applied Mathematics, 58:239-252, 1995.

[24] M. Kutz. Computing shortest non-trivial cycles on orientable surfaces of bounded genus in almost linear time. In SoCG, pages 430-438, 2006.

[25] F. Lazarus, M. Pocchiola, G. Vegter, and A. Verroust. Computing a canonical polygonal schema of an orientable triangulated surface. In $S o C G$, pages $80-89$, 2001.

[26] T. Lewiner, H. Lopes, J. Rossignac, and A. W. Vieira. Efficient edgebreaker for surfaces of arbitrary topology. In Sibgrapi, pages 218-225, 2004.

[27] H. Lopes, J. Rossignac, A. Safonova, A. Szymczak, and G. Tavares. Edgebreaker: a simple implementation for surfaces with handles. Computers E Graphics, 27(4):553-567, 2003.

[28] B. Mohar and C. Thomassen. Graphs on Surfaces. Johns Hopkins, 2001.

[29] D. Poulalhon and G. Schaeffer. Optimal coding and sampling of triangulations. Algorithmica, 46:505-527, 2006.

[30] J. Rossignac. Edgebreaker: Connectivity compression for triangle meshes. Transactions on Visualization and Computer Graphics, 5:47-61, 1999.

[31] W. Schnyder. Planar graphs and poset dimension. Order, pages 323-343, 1989.

[32] W. Schnyder. Embedding planar graphs on the grid. In $S O D A$, pages 138-148, 1990.

[33] G. Turan. On the succint representation of graphs. Discrete Applied Mathematics, 8:289-294, 1984.

[34] W. Tutte. A census of planar maps. Canadian Journal of Mathematics, 15:249-271, 1963.

[35] G. Vegter and C.-K. Yap. Computational complexity of combinatorial surfaces. In SoCG, pages 102-111, 1990 . 